\title{
Impacto de la vacunación universal frente a la varicela en Navarra, 2006-2010
}

\section{Impact of universal varicella vaccination in Navarre, 2006-2010}

\author{
M. García Cenoz, J. Castilla, F. Irisarri, M. Arriazu, A. Barricarte
}

\section{RESUMEN}

Fundamento. En 2007 se introdujo la vacunación universal frente a la varicela en el calendario vacunal de Navarra. Este estudio tiene por objeto evaluar el impacto de dicha medida en la incidencia de varicela tanto en las cohortes vacunadas (efecto directo), como en las no vacunadas (efecto indirecto).

Material y métodos. La varicela es una enfermedad de declaración obligatoria individualizada. Analizamos la incidencia anual por grupos de edad entre 2006 y 2010. Del conjunto mínimo básico de datos al alta hospitalaria se tomaron los ingresos con diagnóstico principal de varicela o de varicela complicada de los años 2006 a 2009.

Resultados. La incidencia de varicela ha disminuido un $93,0 \%$, desde 8,04 casos por 1.000 habitantes en 2006 a 0,56 por 1000 habitantes en $2010(\mathrm{p}<0,0001)$. En niños de 1 a 6 años (cohortes vacunadas), la incidencia de la varicela ha disminuido un $96,3 \%$. En las cohortes vacunadas a los 10 y 14 años, también se observa un descenso del $93,6 \%$ en niños de 10 a 14 años, y de un $85,0 \%$ en los de 15 a 19 años. En los grupos de edad no vacunados observamos descensos del $88,2 \%$ en los niños menores de un año, del $73,3 \%$ en los de 7 a 9 años, y del $84,6 \%$ en personas mayores de 20 años.

En 2006 se produjeron 25 ingresos hospitalarios por varicela en Navarra y en 2009 esta cifra descendió a 7. La tasa de ingresos descendió un $73 \%$.

Conclusión. La introducción de la vacunación universal de la varicela en Navarra ha llevado a una disminución rápida y muy pronunciada de la incidencia de la varicela, tanto en vacunados como en no vacunados.

Palabras clave. Varicela. Vacunación.

\begin{abstract}
Background. In 2007 universal varicella vaccination was introduced in the childhood immunization schedule of of Navarre. The aim of this study is to evaluate the impact of this measure on the incidence of varicella in both vaccinated cohorts (direct effect) and in the unvaccinated (indirect effect).
\end{abstract}

Material and methods. Varicella is a notifiable disease. We analyzed the annual incidence by age groups between 2006 and 2010. Hospital admissions with varicella or complicated varicella as the principal diagnosis were obtained from the minimum basic data set on hospital discharges for the years 2006 to 2009 .

Results. The incidence of varicella has decreased by $93.0 \%$, from 8.04 cases per 1,000 inhabitants in 2006 to 0.56 per 1,000 inhabitants in $2010(\mathrm{p}<0,0001)$. In children from 1 to 6 years (vaccinated cohorts), the incidence of varicella has fallen by $96.3 \%$. In the cohorts vaccinated at 10 and 14 years, a decrease of $93.6 \%$ can also be observed in children from 10 to 14 years, and of $85.0 \%$ in those of 15 to 19 years. In the unvaccinated age groups we can observe falls of $88.2 \%$ in children under one year, of $73.3 \%$ in those of 7 to 9 years, and of $84.6 \%$ in people over 20 years.

In 2006 there were 25 hospital admissions due to varicella in Navarre and in 2009 this figure decreased to 7 . The rate of admissions fell by $73 \%$.

Conclusion. The introduction of universal varicella vaccination in Navarre has resulted in a rapid and very steep reduction of the incidence of varicella in both vaccinated and unvaccinated people.

Key words. Varicella. Vaccination.
Instituto de Salud Pública de Navarra.

Recepción: 10 de marzo de 2011

Aceptación provisional: 6 de abril de 2011

Aceptación definitiva: 13 de mayo de 2011

\author{
Correspondencia \\ Manuel García Cenoz \\ Instituto de Salud Pública \\ Leyre, 15 \\ 31003 Pamplona \\ E-mail.mgcenoz@navarra.es
}




\section{INTRODUCCIÓN}

En ausencia de vacunación el virus de la varicela circula ampliamente, infectando a la mayor parte de la población durante la infancia. En 1995 se introdujo la primera vacuna frente a la varicela en el calendario vacunal infantil de los Estados Unidos y en los años posteriores se observó una reducción, no sólo de los casos de varicela sino también de las hospitalizaciones debidas a sus complicaciones ${ }^{1-3}$. En otros países como Australia ${ }^{4,5}$ o los Países bajos ${ }^{6}$ la introducción de la vacunación universal infantil frente a la varicela ha tenido efectos similares.

En España se introdujo a partir de 2007 la vacunación universal frente a la varicela en el calendario de vacunaciones infantiles de Madrid, Navarra, Ceuta y Melilla.

Con el objetivo de reducir la circulación del virus de la varicela en la población de forma rápida y evitar bolsas de susceptibles, en Navarra se introdujo desde 2007 la vacunación universal de la varicela con una triple estrategia: a los 15 meses, a los 3 años, manteniéndose además la vacunación frente a la de los 10 años de edad para individuos susceptibles ${ }^{7}$. Desde 2009 se ha incorporado además una segunda dosis de acuerdo con la modificación de la ficha técnica de Varivax ${ }^{\circledR}$ por parte de la Agencia Española del Medicamento y Productos Sanitarios ${ }^{8}$.

La vacuna de la varicela, como algunas otras vacunas ejerce no solo un efecto de protección directa sobre las personas vacunadas, sino que también se le atribuye una protección colectiva indirecta al reducir la probabilidad de que los sujetos susceptibles entren en contacto con el virus $^{9,10}$. Este efecto indirecto puede tener un peso relevante en el impacto total de algunos programas de vacunación.

El objetivo del presente estudio es describir la evolución de la incidencia de la varicela en Navarra desde la introducción de la vacunación universal y evaluar el impacto de la vacunación, tanto en las cohortes vacunadas (efecto directo), como en las no vacunadas (efecto indirecto).

\section{MATERIAL Y MÉTODOS}

La varicela es una enfermedad de declaración obligatoria en Navarra desde $1982^{11}$. El sistema de enfermedades de declaración obligatoria recoge el número de casos semanales de varicela, diagnosticados por cada médico. Desde 2005 la historia clínica informatizada está implantada en todos los centros de la red pública de atención primaria de Navarra y su utilización es generalizada. De la historia clínica se han tomado todos los casos con diagnóstico de varicela (Clasificación Internacional de Atención Primaria -CIAP2-, código A72) en los años 2006 a 2010, con información del sexo, edad, zona básica de salud e historia de vacunación.

Los ingresos hospitalarios por varicela se obtuvieron del Conjunto Mínimo Básico de Datos al alta hospitalaria. Se tomaron las altas con diagnóstico principal de varicela (código de la Clasificación Internacional de Enfermedades, novena revisiónmodificación clínica, 052.9) o complicación de varicela (códigos CIE-9 MC: 052.0, 052.1, 052.7, 052.8) de los años 2006 a 2009.

Para el cálculo de tasas de incidencia se ha utilizado la población de Navarra a principio de cada año según el Padrón Municipal que publica el Instituto Nacional de Estadística.

En Navarra la vacunación frente a la varicela se ha ido extendiendo de forma escalonada. En 2004 se inició la vacunación a susceptibles a los 14 años para las cohortes nacidas a partir de 1990. En 2006 se adelantó la vacunación de susceptibles a los 10 años (cohortes nacidas a partir de 1996), y se realizó una campaña de vacunación escolar para los susceptibles nacidos entre 1992 y 1995. En 2007 se inició la vacunación universal con una dosis a los 15 meses para los nacidos en 2006 y se condicionó la aplicación de la segunda dosis a los 3 años a la autorización de la misma por parte de la Agencia Española de Medicamentos y Productos Sanitarios ${ }^{7}$. Además se realizó una campaña de vacunación a los tres años, a los nacidos en 2004 y 2005, manteniéndose la vacunación a suscepti- 
bles a los 10 años, para las cohortes nacidas con anterioridad a 2004 (Tabla 1). Esta vacunación se realiza simultáneamente con la de la triple vírica, y las coberturas alcanzadas con ambas vacunas son elevadas y tienden a ser similares, con la única diferencia que se explica por los niños que llegada la edad de vacunación ya han pasado la varicela y por tanto no se vacunan (96\% para la primera dosis de triple vírica y $88 \%$ para la primera dosis de varicela en el periodo $2007-2008$, y $89 \%$ y $81 \%$ para la segunda dosis de triple vírica y varicela, respectivamente en 2009-2010).

Tabla 1. Vacunación frente a la varicela por cohortes de nacimiento en Navarra hasta 31 diciembre 2010

\begin{tabular}{ccccc}
\hline \multirow{2}{*}{$\begin{array}{c}\text { Cohorte de } \\
\text { nacimiento }\end{array}$} & \multicolumn{2}{c}{ Año vacunación } & \multicolumn{2}{c}{ Edad vacunación } \\
\cline { 2 - 5 } $\mathbf{1}^{\mathbf{a}} \mathbf{d o s i s}$ & $\mathbf{2}^{\mathbf{a}}$ dosis & $\mathbf{1}^{\mathbf{a}}$ dosis & $\mathbf{2}^{\mathbf{a}}$ dosis \\
\hline $1990^{*}$ & 2004 & 2004 & 14 años & 14 años \\
$1991^{*}$ & 2005 & 2005 & 14 años & 14 años \\
1992 & 2006 & 2006 & 14 años & 14 años \\
1993 & 2006 & 2006 & 13 años & 13 años \\
1994 & 2006 & 2010 & 12 años & 16 años \\
1995 & 2006 & 2010 & 11 años & 15 años \\
1996 & 2006 & 2010 & 10 años & 14 años \\
1997 & 2007 & 2010 & 10 años & 13 años \\
1998 & 2008 & 2010 & 10 años & 12 años \\
1999 & 2009 & 2010 & 10 años & 11 años \\
2000 & 2010 & 2010 & 10 años & 10 años \\
$2001-2003$ & & Cohortes no vacunadas & - \\
2004 & 2007 & - & - & - \\
2005 & 2008 & - & - & 3 años \\
2006 & $2007 / 2008$ & 2009 & 15 meses & 3 años \\
2007 & $2008 / 2009$ & 2010 & 15 meses \\
2008 & $2009 / 2010$ & - & 15 meses & 3 años \\
2009 & $2010 / 2011$ & - & 15 meses & \\
\hline
\end{tabular}

*Vacunados con Varilrix ${ }^{\circledR}$. El resto de las cohortes fueron vacunadas con Varivax ${ }^{\circledR}$.

Se evaluó el impacto directo, en los grupos de edad sometidos a vacunación universal, que refleja el efecto individual de la vacunación, y el impacto indirecto, en los grupos de edad no sometidos a vacunación universal.

Para estudiar el impacto de la vacunación frente a la varicela se ha comparado la incidencia en 2006 (año previo a la vacunación universal) y 2010. Para las compara- ciones estadísticas se utilizó la prueba $\chi^{2}$ y la prueba exacta de Fisher.

\section{RESULTADOS}

La figura 1 muestra la serie histórica de casos declarados de varicela en Navarra desde 1980 hasta 2009. Se observa el marcado carácter estacional de la enfermedad, con predominio en los meses de 
invierno y primavera, y un pico máximo hacia la cuatrisemana 21-24. A partir de 2006 , se inicia un cambio del patrón temporal de la enfermedad, con un descenso en la incidencia y una suavización de la estacionalidad con una distribución de casos más homogénea durante los meses de otoño e invierno (Fig. 2).
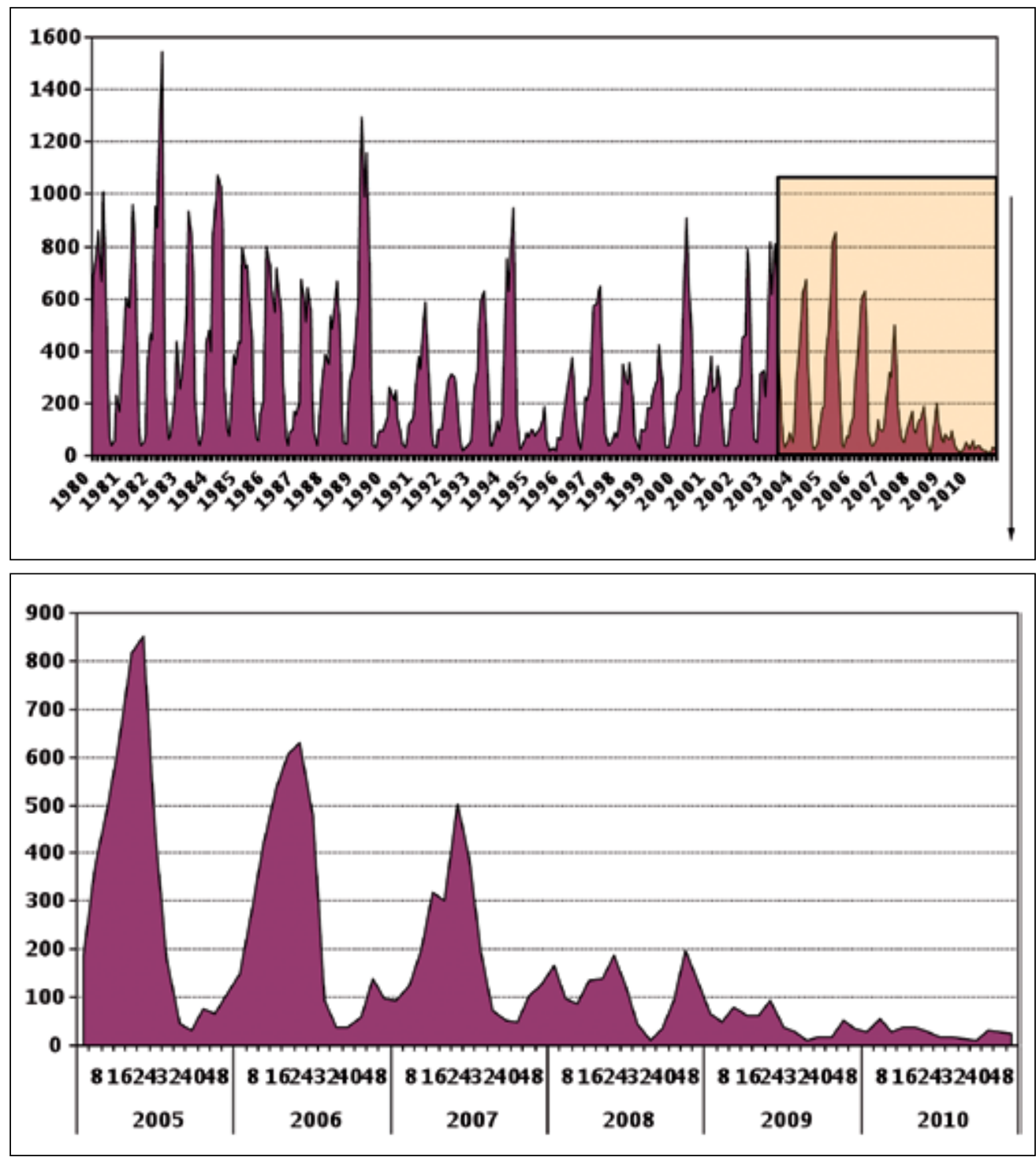

Figura 1. Casos de varicela declarados en Navarra por cuatrisemanas, 1980-2010. Fuente: Sistema de Declaración Obligatoria de Enfermedades de Navarra. 


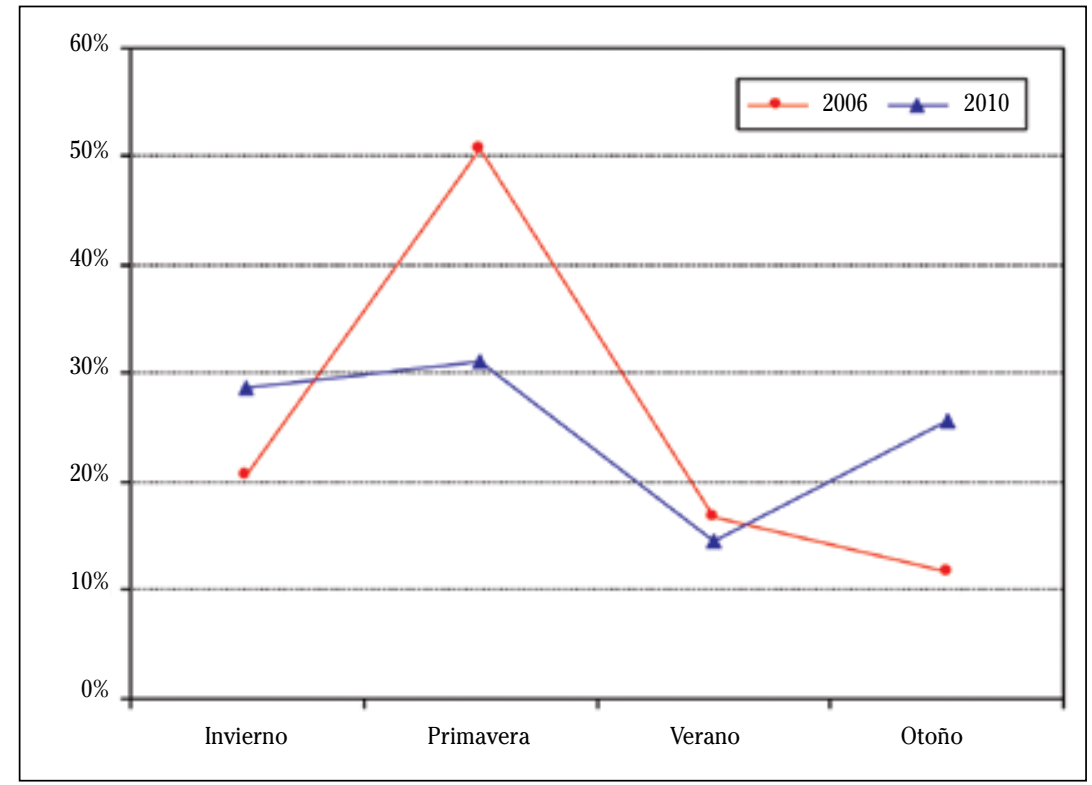

Figura 2. Estacionalidad de los diagnósticos de varicela, 2006-2010.

En el periodo 2006-2010 se diagnosticaron 10.062 casos de varicela en atención primaria. El 50,7\% de los casos se produjeron en hombres. El 63,3\% de los casos se diagnosticaron en menores de 5 años, y el $88,2 \%$ en menores de 15 años. La incidencia de varicela ha disminuido progresivamente

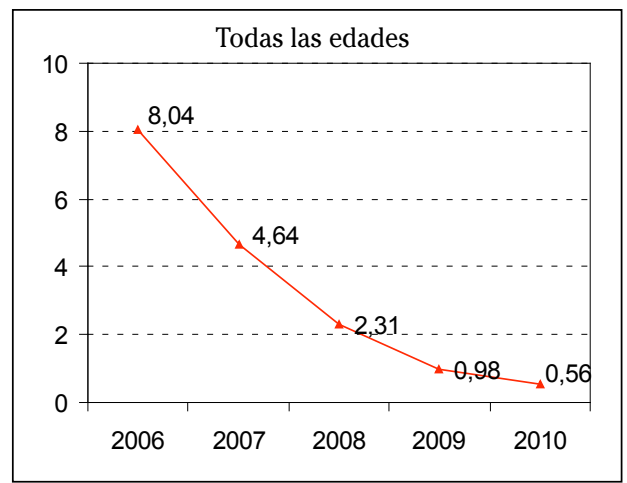

en los últimos años, pasando de 8,04 casos por 1.000 habitantes en 2006, a 0,56 casos por 1.000 habitantes en 2010 , lo cual supone una reducción del 93,0\% ( $<<0,0001)$ (Fig. 3 ). Esta reducción ha sido del $94,3 \%$ en los menores de 15 años y del 82,9\% en las personas de 15 o más años.

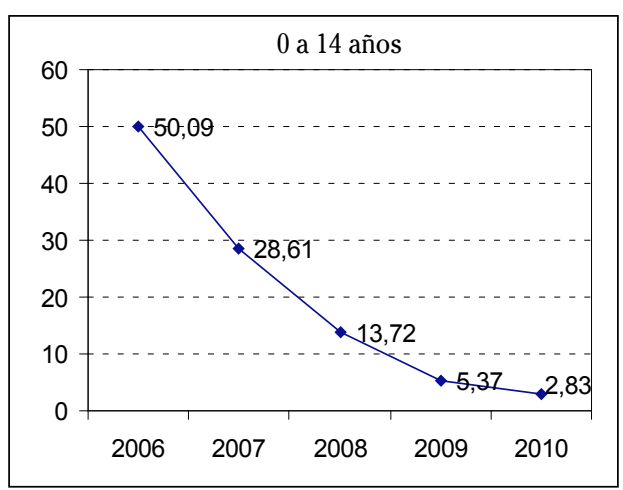

Figura 3. Tasas de incidencia anual de varicela por 1000 habitantes en Navarra, 2006-2010. 
La edad del diagnóstico de la varicela se ha retrasado. Así, mientras que en 2006 el $89,2 \%$ de los casos se diagnosticaban en menores de 15 años, en 2010 se diagnostican el 76,6\% de los casos totales. Del mismo modo, va disminuyendo el pico de incidencia de varicela en los niños de tres años. En su lugar, en 2010, el mayor número de casos se produjeron a los 7 años de edad, y que coincide con la última cohorte que todavía no ha sido vacunada (Fig. 4).

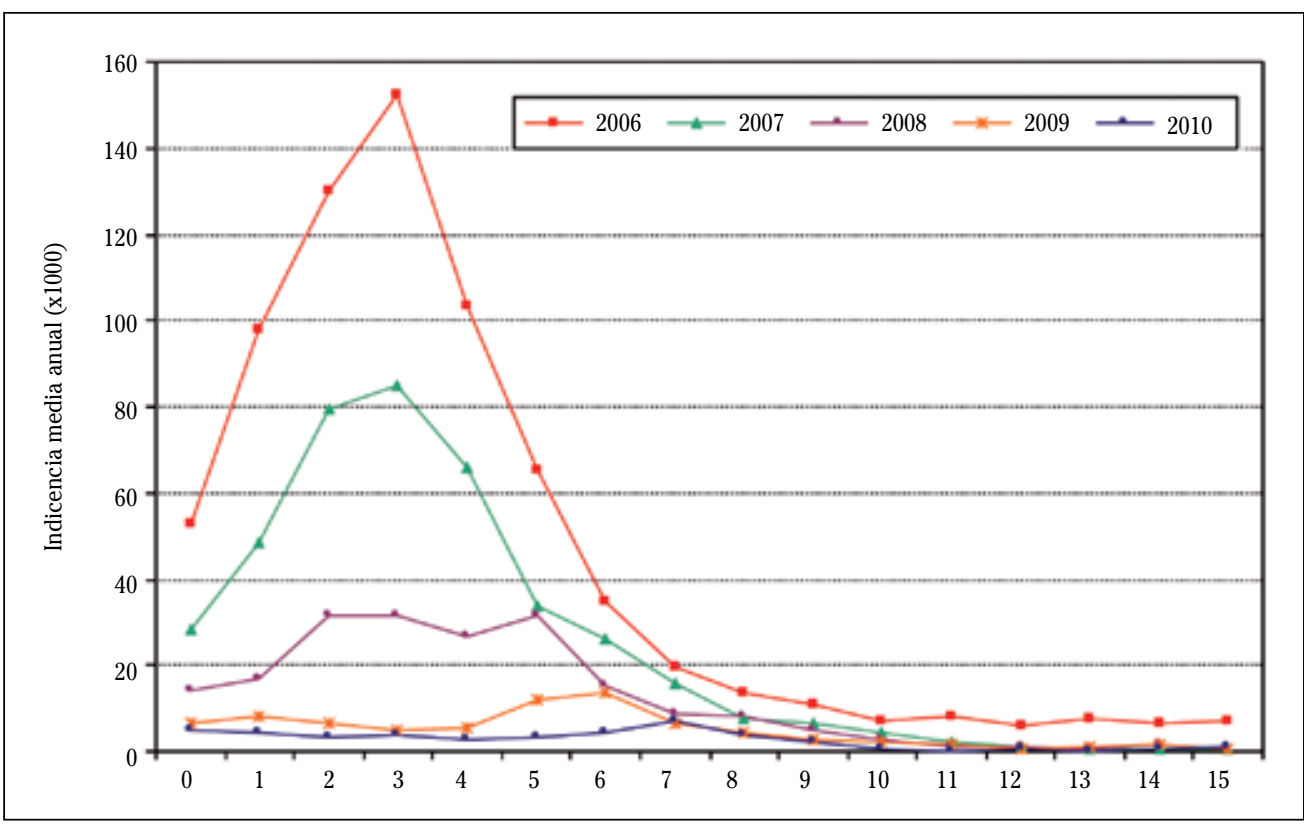

Figura 4. Incidencia anual de varicela por 1.000 habitantes en población menor de 15 años, según grupos de edad año a año, 2006-2010.

En niños de 1 a 6 años, donde se incluirían las cohortes vacunadas a partir del inicio de la vacunación universal, la incidencia de la varicela ha disminuido en conjunto un $96,3 \%$. Los descensos son igualmente importantes en todos los grupos de edad anual de 1 a 6 años. En niños y adolescentes de 10 a 19 años, que incluyen las cohortes vacunadas a los 10 o a los 14 años, también se observa una importante disminución de la incidencia de la varicela, con un descenso del 93,6\% entre 2006 y 2010 en niños de 10 a 14 años, y de un $85,0 \%$ en los de 15 a 19 años (Tabla 2). 
Tabla 2. Efecto directo de la vacunación universal frente a la varicela en niños de 1 a 6 años y de la vacunación de susceptibles a los 10 y 14 años

\begin{tabular}{|c|c|c|c|c|c|c|c|}
\hline & \multicolumn{5}{|c|}{ Incidencia (x 1.000) } & \multirow{2}{*}{$\begin{array}{l}\text { Reducción } \\
\text { 2006-2010 }\end{array}$} & \multirow{2}{*}{$\mathbf{p}$} \\
\hline & 2006 & 2007 & 2008 & 2009 & 2010 & & \\
\hline \multicolumn{8}{|c|}{ Vacunación universal } \\
\hline 1 año & 70 & 33 & 19 & 8 & 5 & $92,4 \%$ & $<0,0001$ \\
\hline 2 años & 118 & 67 & 11 & 7 & 4 & $96,8 \%$ & $<0,0001$ \\
\hline 3 años & 133 & 78 & 47 & 5 & 4 & $97,3 \%$ & $<0,0001$ \\
\hline 4 años & 143 & 86 & 15 & 5 & 3 & $97,7 \%$ & $<0,0001$ \\
\hline 5 años & 95 & 49 & 43 & 4 & 3 & $97,0 \%$ & $<0,0001$ \\
\hline 6 años & 52 & 30 & 21 & 22 & 4 & $93,2 \%$ & $<0,0001$ \\
\hline Total 1-6 años & 102 & 58 & 26 & 9 & 4 & $96,3 \%$ & $<0,0001$ \\
\hline \multicolumn{8}{|c|}{ Vacunación de susceptibles } \\
\hline 10-14 años & 7,8 & 2,5 & 1,9 & 1,6 & 0,5 & $93,6 \%$ & $<0,0001$ \\
\hline 15-19 años & 3,9 & 1,1 & 0,6 & 0,7 & 0,6 & $85,0 \%$ & $<0,0001$ \\
\hline
\end{tabular}

En los grupos de edad no vacunados se observa también una importante disminución de la incidencia de varicela entre 2006 y 2010 , con descensos del $88,2 \%$ en los niños menores de un año, del $77,9 \%$ en los de 7 años, del $59,2 \%$ en los de 8 años, y del $79,5 \%$ en los de 9 años. En las personas mayores de 20 años, esta disminución fue del $84,6 \%$ (Tabla 3 ).

Tabla 3. Efecto indirecto de la vacunación universal frente a la varicela. Reducción de la incidencia de la varicela en las cohortes de nacimiento no vacunadas (niños menores de 1 año y de 7 a 9 años, y adultos de 20 o más años)

\begin{tabular}{cccccccc}
\hline & \multicolumn{9}{c}{ Incidencia (x 1.000) } & Reducción & p \\
\cline { 2 - 6 } & $\mathbf{2 0 0 6}$ & $\mathbf{2 0 0 7}$ & $\mathbf{2 0 0 8}$ & $\mathbf{2 0 0 9}$ & $\mathbf{2 0 1 0}$ & $\mathbf{2 0 0 6 - 2 0 1 0}$ & \\
\hline$<1$ año & 13 & 9 & 5 & 2 & 1 & $88,2 \%$ & $<0,0001$ \\
7 años & 28 & 18 & 9 & 7 & 6 & $77,9 \%$ & $<0,0001$ \\
8 años & 14 & 12 & 10 & 5 & 6 & $59,2 \%$ & $<0,0001$ \\
9 años & 12 & 6 & 6 & 3 & 2 & $79,5 \%$ & $<0,0001$ \\
Subtotal de 7-9 años & 18 & 13 & 8 & 5 & 5 & $73,3 \%$ & $<0,0001$ \\
$20-24$ años & 2,0 & 1,3 & 0,6 & 0,5 & 0,3 & $83,9 \%$ & $<0,0001$ \\
$25-44$ años & 1,6 & 0,9 & 0,6 & 0,3 & 0,2 & $85,2 \%$ & $<0,0001$ \\
$45-64$ años & 0,2 & 0,2 & 0,1 & 0,08 & 0,06 & $76,0 \%$ & 0,0001 \\
$\geq 65$ años & 0,1 & 0,07 & 0,04 & 0,03 & 0,02 & $82,7 \%$ & 0,0207 \\
Subtotal de $\geq \mathbf{2 0}$ años & 0,9 & 0,5 & 0,3 & 0,2 & 0,1 & $84,6 \%$ & $<0,0001$ \\
\hline
\end{tabular}


En 2006 se produjeron 25 ingresos hospitalarios por varicela en Navarra y en 2009 esta cifra descendió a 7. La tasa de ingresos descendió un $73 \%$ en este periodo. La estancia media de los ingresos no varió sig- nificativamente entre ambos periodos. En ambos periodos el porcentaje de varicela complicada fue similar (40,0\% de los casos en 2006 y $42,9 \%$ en 2009) (Tabla 4).

Tabla 4. Casos con ingreso hospitalario con diagnóstico principal de varicela (052.9) o complicación de varicela (códigos CIE-9_MC: 052.0, 052.1, 052.7, 052.8)

\begin{tabular}{lccccc}
\hline & $\mathbf{2 0 0 6}$ & $\mathbf{2 0 0 7}$ & $\mathbf{2 0 0 8}$ & $\mathbf{2 0 0 9}$ & $\begin{array}{c}\text { Reducción } \\
\mathbf{2 0 0 6}-2009\end{array}$ \\
\hline Ingresos hospitalarios & 25 & 22 & 11 & 7 & $72 \%$ \\
\hline Estancia media en días & 5,9 & 5,0 & 3,4 & 5,0 & \\
\hline Tasa de ingresos por 100.000 hab. & 4,2 & 3,6 & 1,8 & 1,1 & $73 \%{ }^{*}$ \\
\hline $\begin{array}{l}\text { Casos de varicela complicada, } \\
\text { \% de casos hospitalizados }\end{array}$ & $40,0 \%$ & $45,5 \%$ & $18,2 \%$ & $42,9 \%$ & \\
\hline
\end{tabular}

*Comparación de tasas de 2006 y 2009, p<0,0001

\section{DISCUSIÓN}

Antes de la vacunación universal la varicela en Navarra era una enfermedad de alta incidencia, más frecuente en los meses de invierno y primavera, y con tendencia a originar brotes. La máxima incidencia se registraba entre los 2 y 3 años de edad; el $75 \%$ de los casos se producía antes de los 6 años y el $90 \%$ antes de los 15 años $^{12}$.

La incorporación de la vacunación universal frente a la varicela en el calendario de vacunaciones de Navarra está motivando una serie de cambios en la presentación de esta enfermedad, con un progresivo cambio del patrón temporal de la enfermedad. De acuerdo a lo publicado por otros autores ${ }^{13}$, hemos observado cómo en los últimos años está disminuyendo paulatinamente el pico de incidencia que, en nuestro caso, venía ocurriendo entre las semanas 20 y 24, pasando a una distribución de los casos anuales más homogénea durante los meses de otoño e invierno. De forma similar, otro estudio observa un paulatino aplanamiento de los picos primaverales de varicela ${ }^{14}$.

El principal aspecto a destacar es la elevada disminución, del 93\%, de la incidencia de varicela en el conjunto de la población. Ésta es especialmente pronunciada en las cohortes vacunadas, principalmente en los niños de 1 a 6 años, con reducciones mayores del $95 \%$, superiores a lo comunicado en otros lugares donde también se introdujo la vacunación universal ${ }^{3,14-16}$. Así mismo, hemos observado una importante disminución de la incidencia en población de 10 a 19 años, derivada de la estrategia de vacunación a susceptibles.

Destaca también que en las cohortes que no se han beneficiado de la vacunación universal ha disminuido la incidencia de varicela de forma considerable, y muy especialmente en los niños menores de un año $(88,2 \%)$, lo que se atribuye al efecto de la inmunidad colectiva o de rebaño, motivada por una disminución de la circulación del virus de la varicela ${ }^{10}$. En otros grupos etáreos (niños entre 7 y 9 años y población mayor de 20 años), la disminución es también significativa, si bien en los adultos de mayor edad, el impacto es menor dada la baja incidencia que ya tenía esta enfermedad previamente.

Todos estos cambios conllevan un ligero desplazamiento del patrón etáreo de la enfermedad. La proporción de casos me- 
nores de 15 años ha disminuido desde el $89,2 \%$ en 2006 al $76,6 \%$ en 2010 , y el pico de máxima incidencia se desplaza de los 3 a los 7 años de edad, debido a que esa cohorte, está formada por niños que todavía no se han beneficiado de la vacunación universal frente a la varicela. Por tanto, es previsible que conforme se extienda la implantación de la vacunación a todas las cohortes de niños, este fenómeno desaparezca.

La disminución de la incidencia de la varicela en la población se acompaña de una disminución del $73 \%$ de la tasa de ingresos hospitalarios por varicela, que coincide con lo observado en otros lugares ${ }^{2,13,17}$. Este cambio no parece haberse acompañado de una mayor gravedad, como lo demuestra el hecho de que tanto la duración de la hospitalización, como los casos de varicela complicados se mantengan en valores similares.

En conclusión, aunque sólo disponemos de una serie de cuatro años desde el inicio de la vacunación universal frente a la varicela, nuestros resultados muestran que la introducción de la vacunación universal de la varicela en Navarra, ha llevado a una disminución rápida y muy pronunciada de la incidencia de la varicela, tanto en vacunados como en no vacunados. En esta situación es necesario mantener altas coberturas y continuar con la estrategia de vacunación de susceptibles al inicio de la adolescencia para evitar la acumulación de personas susceptibles que pudiera dar lugar a brotes de enfermedad en edades más tardías, y por consiguiente aumentar las formas graves de la enfermedad.

\section{BIBLIOGRAFÍA}

1. Seward JF, Watson BM, Peterson Cl, Mascola L, Pelosi JW, Zhang JX et al. Varicella disease after introduction of varicella vaccine in the United States, 1995-2000. JAMA 2002; 287: 606-611.

2. Grose C. Varicella vaccination of children in the United States: Assessment after the first decade 1995-2005. J Clin Virol 2005; 33: 89-95.

3. Seward JF, Marin M, Vazquez M. Varicella vaccine effectiveness in the US vaccination program: A review. J Infect Dis 2008; 197: S82-S9.
4. Macartney KK, Beutels P, McIntyre P, Burgess MA. Varicella vaccination in Australia. J Paediatr Child Health 2005; 41: 544-552.

5. Carville KS, Riddell MA, Kelly HA. A decline in varicella but an uncertain impact on zoster following varicella vaccination in Victoria, Australia. Vaccine 2010; 28: 2532-2538.

6. Boot HJ, de Melker He, Stolk EA, de Wit GA, KImman TG. Assessing the introduction of universal varicella vaccination in the Netherlands. Vaccine 2006; 24: 6288-6299.

7. Boletín Oficial de Navarra. Orden Foral $133 / 2006$, de 13 de diciembre, de la Consejería de Salud, por la que se modifica en Calendario Oficial de Vacunaciones de Navarra. BON núm 4, 08/01/2007. Boletín Oficial de Navarra 2007.

8. Agencia española de medicamentos y productos sanitarios. Ficha técnica de Varivax®, revisión 2008. Available from: https:// sinaem4.agemed.es/consaem/especialidad. do? metodo=verFichaWordPdf \&codigo $=6570$ $9 \&$ formato=pdf\&formulario=FICHAS

9. Fox J, Elveback L, Scott W, Gatewood L, AckerMAN E. Herd immunity: basic concept end relevance to public health immunization practices. Am J Epidemiol 1971; 94: 179-189.

10. Fox J. Herd immunity and measles. Rev Infect Dis 1983; 5: 463-464.

11. Boletín Oficial de Navarra. Orden Foral 19/2008, de 15 de febrero, de la Consejería de Salud, por la que se modifican las enfermedades incluidas en el Sistema de Vigilancia Epidemiológica de Navarra. BON núm 28, 01/03/2008. 2008.

12. García Cenoz M, Castilla J, Montes Y, Moran J, SAlaberri A, Elia F et al. Varicella and herpes zoster incidence prior to the introduction of systematic child vaccination in Navarre, 2005-2006. An Sist Sanit Navar 2008; 31: 7180 .

13. Kwong JC, Tanuseputro P, Zagorski B, Moineddin $\mathrm{R}$, CHAN KJ. Impact of varicella vaccination on health care outcomes in Ontario, Canada: Effect of a publicly funded program? Vaccine 2008; 26: 6006-6012.

14. SiEdLER A, ARndt U. Impact of the routine varicella vaccination programme on varicella epidemiology in Germany. Euro Surveill 2010; 15 (13):pii=19530.

15. Mullooly JP, Maher JE, Drew L, Schuler R, Hu W. Evaluation of the impact of an HMO's varicella vaccination program on incidence of varicella. Vaccine 2004; 22: 1480-1485. 
16. KUPEK E, TRITANY EF. Impact of vaccination against varicella on the reduction of the disease incidence in children and adolescents from Florianopolis, Brazil. J Pediatr (Rio J) 2009; 85: 365-368.
17. Davis MM, Patel MS, Gebremariam A. Decline in varicella-related hospitalizations and expenditures for children and adults after introduction of varicella vaccine in the United States. Pediatrics 2004; 114: 786-792. 